\title{
Effects of a Systems-Level Intervention to Improve Trainer Integrity in a Behavioral Healthcare Organization
}

\author{
Abigail L. Blackman ${ }^{1} \cdot$ Sandra A. Ruby ${ }^{1} \cdot$ Grace Bartle $^{1} \cdot$ Florence D. DiGennaro Reed $^{1} \cdot$ Michael Strouse $^{2}$. \\ Tyler G. Erath ${ }^{1} \cdot$ Marren Leon-Barajas ${ }^{1,2}$
}

Accepted: 5 February 2022 / Published online: 2 March 2022

(c) The Author(s), under exclusive licence to Springer Nature Switzerland AG 2022

\begin{abstract}
Objectives Direct support professionals (DSPs) play a critical role in health-related outcomes for individuals with intellectual and developmental disabilities (IDD) who reside in congregate living settings. Large behavioral healthcare organizations often rely on staff to function as peer trainers for newly hired DSPs. Organizations should adopt empirically supported training techniques to prepare peer trainers for their role and develop systems to ensure ongoing integrity of the training system. The purpose of this program description is to summarize consultation activities that attempted to create these systems.

Methods Staff members were trained to function as peer trainers, an assessment was conducted to determine the barriers to training in the natural environment, and a systems-level intervention informed by the assessment was implemented to improve peer trainer integrity.

Results The assessment revealed peer trainers were often unaware when they were expected to train and did not receive feedback or programmed consequences for training newly hired DSPs. A systems-level intervention containing a prompt (reminder) about upcoming training and feedback plus a monetary incentive produced improvements in trainer integrity.

Conclusions A systems-level intervention based on an assessment can improve peer trainer integrity. Ensuring peer trainer integrity increases the likelihood that newly hired DSPs will implement health-related protocols with individuals with IDD.
\end{abstract}

Keyword Systems-level intervention · Staff training · Performance management · Trainer integrity $\cdot$ Incentive ·

Performance diagnostic checklist

Currently, in the USA, there are 7.38 million individuals diagnosed with an intellectual or developmental disability (IDD; Residential Information Systems Project, 2020). Approximately 3.5 million individuals live with family caregivers, including $24 \%$ for whom caregivers are over the age of 60 (Braddock et al., 2013). Nearly 713,300 individuals receive residential support in congregate living settings (Harris-Kojetin et al., 2013), with an additional 473,000 individuals with IDD on waitlists for residential services (Diament, 2020). These numbers are expected to increase (Ryan et al., 2014), which exacerbates existing pressures facing service providers to deliver high-quality

Florence D. DiGennaro Reed

fdreed@ku.edu

1 Department of Applied Behavioral Science, University of Kansas, 4001 Dole Human Development Center, 1000 Sunnyside Avenue, Lawrence, KS 66045-7555, USA

2 GoodLife Innovations, Inc, Lenexa, KS, USA services in a timely and safe manner (Strouse \& DiGennaro Reed, 2021).

Research has shown that individuals with IDD are at an increased risk of experiencing poor overall health and chronic health conditions (Centers for Disease Control \& Prevention, 2020). For example, adults with IDD are at high risk for obesity and associated secondary conditions, including cardiovascular disease, diabetes, and hypertension (Anderson et al., 2013; Tyler et al., 2011). The coronavirus disease of 2019 (COVID-19) pandemic revealed that people residing in congregate living settings, specifically those with IDD, are at an increased risk for healthrelated issues including a COVID-19 diagnosis (Armitage \& Nellums, 2020; Gleason et al., 2021). Moreover, individuals with IDD have a decreased life expectancy due to various factors including complications associated with their disability, obstacles related to receiving adequate health care, and socioeconomic status (Gleason et al., 2021). 
Direct support professionals (DSPs) play a critical role in health-related outcomes for individuals with IDD who reside in congregate living settings. An estimated 1.3 million DSPs work with individuals with IDD (President's Committee for People with IDD, 2017). The primary job duties of DSPs include assisting individuals with completing daily living skills (e.g., preparing healthy meals, personal hygiene, laundry) and engaging with others in their community (Friedman, 2019), and providing active treatment, such as teaching valued skills based on individualized or person-centered support plans. Other duties include administering medications and adhering to medication and treatment protocols with nursing supports (Kansas Department for Aging \& Disability Services, 2010). Thus, ensuring DSPs are adequately prepared to perform their job duties is a critical component to achieving desired outcomes for individuals with IDD (Novak et al., 2019).

Given their responsibilities, DSPs require training on a wide range of health-related topics including, but not limited to, how to implement person-centered support plans, ensure individualized diets are followed, use lifts or other adaptive equipment, follow emergency preparedness plans, and administer medications. One approach adopted by service providers with geographically distributed residential programs is to rely on residential staff to function as peer trainers to newly hired DSPs (Luiselli, 2015). A peer training approach may be advantageous for several reasons. First, peer training is relatively affordable in that organizations would likely incur additional costs if a credentialed, professional trainer were responsible for providing one-on-one training for each new hire (DiGennaro Reed et al., 2013). Second, on-site peer trainers have an ongoing presence in the residential program, which could foster maintenance of DSP skills (Demchak et al., 1992; Parsons et al., 2013). Finally, training others may help minimize performance drift of peer trainers and foster maintenance of the very skills they teach (Parsons et al., 2013; Van den Pol et al., 1983).

Although evidence suggests that peer training can be an effective approach to supporting staff (e.g., Green \& Reid, 1994; Harchik et al., 2001), organizations must prepare peer trainers for this important job responsibility and ensure ongoing integrity of the training system. A small number of studies have addressed this issue. For example, Parsons et al. (2013) evaluated the effects of a 60-min group training on trainer integrity (i.e., the extent to which peer trainers accurately implement behavioral skills training [BST]). The group training incorporated components of BST-instructions, modeling, practice, and feedback - to teach peer trainers how to train colleagues two target skills (i.e., embedded teaching, conducting a preference assessment). Using a multiple-probe design, Parsons et al. (2013) documented improvements in trainer integrity during simulated role plays. In addition, BST integrity was $100 \%$ for nearly all trainers during an on-the-job assessment following training. Although these findings documented that organizations can effectively prepare peer trainers, the researchers measured trainer integrity across only two target skills and did not assess generalization or maintenance.

Erath et al. (2020) extended Parsons et al. (2013) by evaluating the effects of a group workshop on trainer integrity. Peer trainers (i.e., residential supervisors, DSPs) employed by a large behavioral healthcare organization were taught to use BST to train confederate staff how to reinforce a desirable behavior during a role-play context. Like Parsons et al. (2013), the group workshop incorporated components of BST. Following the workshop, trainer integrity increased to mastery levels for 10 of 25 participants; 10 additional participants reached mastery with supplemental experimenter feedback. Moreover, training generalized to a novel skill (i.e., presenting choice) and maintained for up to 6 weeks with all three participants for whom follow-up assessments were conducted.

In a follow-up study, Erath et al. (2021) evaluated the effects of video-based training on BST integrity. Four prospective peer trainers were taught to use BST during a role play to teach confederate staff two skills, how to reinforce a desirable behavior and provide choice to consumers. The 13-min video module included on-screen text (i.e., written instruction), voiceover narration (i.e., vocal instruction), and two modeled exemplars (i.e., modeling). Participants completed guided notes while watching the module. The video-based training increased BST integrity to mastery levels for two participants; two additional participants required supplemental experimenter feedback to reach mastery. In addition, participant use of BST generalized to two novel skills (i.e., having positive interactions with consumers, delivering effective instructions), and their performance maintained at 7- to 26-day follow-up probes.

Although effective as an initial effort to improve trainer integrity, Erath et al. $(2020,2021)$ did not measure peer trainer integrity in the workplace amidst real-world servicedelivery challenges. In addition, the training provided to peer trainers addressed only a handful of skills trainers would be expected to teach newly hired staff. We sought to address the limitations of both studies and extend their initial findings by evaluating training integrity within a peer training system that also included ongoing support. The purpose of this program description is to summarize how a consultation model was used to (a) adopt procedures from Erath et al. (2021) to prepare peer trainers for their role, (b) conduct an assessment to determine the variables influencing trainer integrity in the workplace, and (c) implement a systems-level intervention to promote high trainer integrity. Peer trainers were expected to teach newly hired staff over 60 skills, including daily routines, schedules, and health needs; teaching skills and promoting consumer participation; behavior support plans; and professional skills and general information. Our 
program description contains data spanning 3 years across 36 residential and 3 day-service programs with nearly 100 peer trainers. In addition, data were collected before and during the COVID-19 pandemic; thus, modifications to the intervention were necessary to ensure safety.

\section{Method}

\section{Participants}

Ninety-nine employees who worked across 36 residential and 3 day-service programs at a behavioral healthcare organization that served adults with IDD in the midwestern USA were participants (hereafter, peer trainers). The organization required employees to be at least 18 years of age, have a high-school diploma or general equivalence degree, and pass a background check. Peer trainers included DSPs and management staff (e.g., group-home managers). Demographic information for peer trainers is unavailable because we collected data as part of an ongoing consultation arrangement between 2018 and 2021 (i.e., not as a formal research study) and many peer trainers are no longer employed by the organization. The Human Rights Committee at the employer organization and the Human Research and Protection Program at the university approved data collection as part of this consultation arrangement.

Prospective peer trainers were required to participate in a workshop led by the consultation team during which they were taught how to use BST to train newly hired DSPs. Workshops were held in a conference room located at the organization's main office and included between two and 10 peer trainers.
The content included an overview of the responsibilities of a peer trainer, a review of the organization's training checklist detailing the target skills peer trainers were expected to teach and the training components they should use (see https://osf.io/ $\mathrm{j} 7 \mathrm{fdg} /$ ), video-based training (i.e., instructions and video models; Erath et al., 2021), and role play and feedback. All peer trainers were required to implement BST with $100 \%$ accuracy during a role play. In addition, peer trainers received information about the behaviors they were expected to perform during peer training (see "Measures" and "Data Analysis" sections) and were informed that the consultation team would conduct observations in their respective workplaces.

\section{Procedures}

Table 1 summarizes the most relevant consultation activities that took place between Fall 2018 and Fall 2021. A pre-intervention assessment was completed before implementing the systems-level intervention to aid in designing intervention components. Subsequently, the effect of the systems-level intervention on peer trainer performance was evaluated across several phases: baseline, feedback, program announcement, monetary incentive plus feedback, and monetary incentive plus feedback and a prompt.

\section{Setting}

Peer trainers conducted 16 to $24 \mathrm{~h}$ of training with newly hired DSPs in the residential and day-service programs. The residential programs were community homes with a shared living room, dining room, kitchen, and at least one bathroom

Table 1 A timeline of consultation activities

\begin{tabular}{ll}
\hline $\begin{array}{l}\text { Sep-Nov } \\
\text { Se19 }\end{array}$ & Conduct pre-intervention assessment \\
Jan & Summarize assessment results and propose recommendations to the CEO \\
Jan-Sep & Ongoing discussions with the CEO and organizational leaders about potential intervention components and systems change \\
June-Sep & Process change for scheduling peer training and notifying relevant parties; develop measurement system for peer trainer integrity \\
Early Sep & Conduct a single baseline probe to confirm peer training integrity remained low before introducing intervention \\
Late Sep & Received approval from the CEO for the systems-level intervention \\
Oct-Dec & Introduce feedback \\
2020 & \\
Jan-Feb & Program announcement; creation of the individualized peer trainer incentive tracking spreadsheets \\
Late Feb & Introduce the monetary incentive plus feedback \\
Mar-July & Intervention put on hold due to the COVID-19 pandemic \\
Late July & Conduct a single baseline probe to confirm peer training integrity remained low before reintroducing intervention \\
Aug-Nov & Reintroduce the monetary incentive plus feedback \\
Nov-Dec & Add a supplemental prompt to monetary incentive plus feedback \\
2021 & \\
Jan-Sep & Continue monetary incentive plus feedback (intervention remains in effect; selected arbitrary date for data presented on the figure)
\end{tabular}


as well as private bedrooms for each consumer. The dayservice programs had shared art, sensory, and game rooms; a kitchen; and office space. Prior to participating in peer training, newly hired DSPs completed approximately $45 \mathrm{~h}$ of training as part of the organization's initial staff training. The training topics included medication administration, CPR, First Aid, Safety Care training, and behavioral teaching, among other topics.

\section{Observations}

Consultants had access to software (e.g., Humanity) used by the organization, which allowed the team to easily determine where and when to schedule observations. The consultation team arranged an unannounced 30-min observation of each peer trainer for each DSP they trained. Observations were scheduled when the training occurred and took place in-person and remotely. During in-person observations, one member of the consultation team visited the programs where training was scheduled. The observation began when the consultant arrived at the residential or day-service program. Remote observations took place by viewing recorded footage captured via iLink Support Technologies ${ }^{\circledR}$ (DiGennaro Reed \& Reed, 2013; Strouse \& DiGennaro Reed, 2021), with video data saved to a HIPAA-compliant server managed by the organization. The observation began once the consultant reviewed the saved footage to identify a time when the peer trainer and DSP were both present. Remote observations were conducted for residential programs only given software capabilities.

Prior to the start of observations, consultants were trained to collect data on peer trainer behavior when training newly hired DSPs. Training consisted of BST for how to use iLink Support Technologies®; a review of the components of BST and the peer trainer behavior being measured, including exemplars and non-exemplars; a review of the training checklist peer trainers were expected to complete; and data collection practice and feedback. During training, consultants were required to accurately record data for two consecutive peer trainings before conducting observations independently.

\section{Pre-Intervention Assessment}

The pre-intervention assessment was conducted in 2018 and included a small sample of unannounced observations completed via iLink Support Technologies ${ }^{\circledR}$ and an interview. The purpose of the observations was to determine the extent to which peer trainers used BST to train newly hired DSPs. At this point in our consultation, we had not yet identified the three target behaviors that comprised the primary dependent variable (see Table 2). Twenty-three observations occurred at four residential programs. Average BST integrity across observations was 5.3\% (range, $0-40 \%$ ); BST integrity was $0 \%$ for 19 of 23 observations.

Because we observed low levels of training integrity, the Performance Diagnostic Checklist - Human Services (PDCHS; Carr et al., 2013) was conducted to determine the barriers to training newly hired DSPs. The PDC-HS is an indirect assessment tool comprised of 20 "yes" or "no" questions asked in a semi-structured interview format. The questions span four areas: training; task clarification and prompting; resources, materials, and processes; and performance consequences, effort, and competition. A "no" indicates a potential area for improvement. Thus, sections of the assessment with high percentages of questions scored as "no" would be considered areas that require intervention.

We conducted the PDC-HS with four peer trainers at the organization. The assessment was completed as part of a graduate course in organizational behavior management under the supervision of the fourth author. Although an ideal assessment would incorporate all peer trainers, the limited resources available and conflicting schedules made that goal difficult. Figure 1 displays the results of the assessment. The percentage of questions with a "no" response was high in two areas: (a) task clarification and prompting; and (b) performance consequences, effort, and competition. Peer trainers revealed that they did not receive reminders for when they were scheduled to train newly hired DSPs and, thus, were often unprepared for training when DSPs arrived at the workplace. With respect to consequences, three peer trainers revealed that they were not directly monitored by a supervisor and did not receive feedback about the training they provided to newly hired DSPs.

Given these findings, the consultation team prepared a written report for the Chief Executive Officer (CEO) in January 2019 that provided recommended changes to the peer training program. We proposed four changes: (a) process

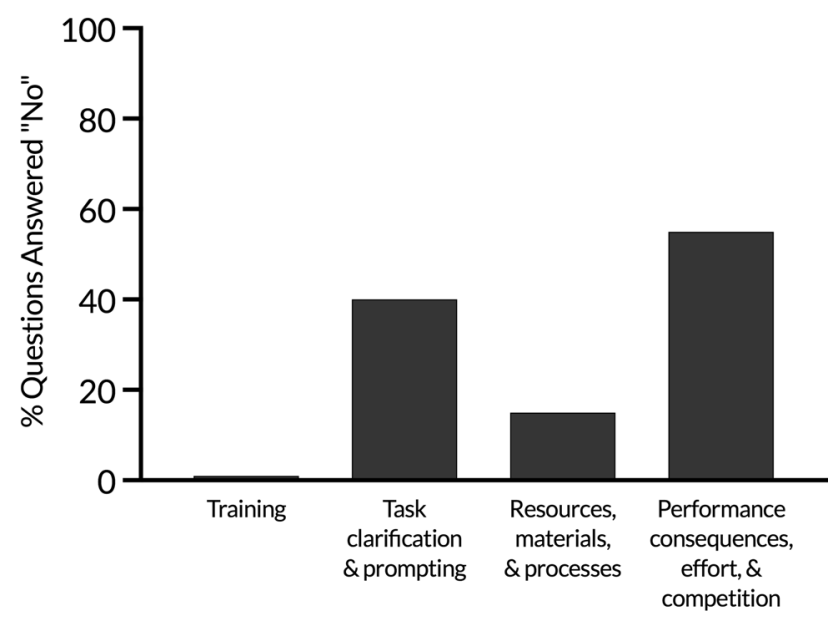

Fig. 1 Pre-intervention assessment results 
improvements so peer trainers or their supervisors were notified in advance when they were scheduled to train; (b) a prompt or reminder to be delivered $24 \mathrm{~h}$ before the scheduled training; (c) supervisor observations of peer training and contingent feedback; and (d) delivery of a monetary incentive to peer trainers for providing training with high integrity. In Summer 2019, while the consultation team was engaged in discussions with the CEO and other organizational leaders about the specific components of the intervention, we assisted the organization with modifying the process by which peer trainers were scheduled to train and how this information was communicated. Given resource constraints, the CEO and consultation team agreed that (a) the consultation team would conduct observations and deliver feedback to peer trainers, and (b) the organization would wait to implement the monetary incentive until collecting data on the efficacy of feedback alone.

\section{Baseline}

Prior to baseline, peer trainers had participated in the workshop, successfully implemented BST during a role play with a consultant, received information about the organization's expectations for peer trainer integrity, and were informed that consultants would conduct unannounced observations in their workplace. During baseline, peer trainers did not experience any programmed antecedents or consequences for conducting peer training as intended.

\section{Feedback}

During the feedback phase, consultants delivered feedback to peer trainers immediately following each observation. The feedback included praise for behaviors performed correctly (e.g., "Excellent job using BST during training. I like how you provided helpful feedback to the staff.") and supportive feedback for behaviors requiring improvement (e.g., "Your professional conduct didn't meet expectations."). In addition, consultants described how peer trainers could improve performance when errors were made (e.g., "Please avoid saying negative comments about your supervisor to new staff."). Peer trainers had the opportunity to ask questions and problem-solve training issues they were experiencing after feedback was delivered.

\section{Program Announcement}

During the program announcement phase, the organization shared details with employees about the systems-level intervention. The period spanned approximately 6 weeks during which peer trainers received an email from the Human Resources Department describing the monetary incentive (summarized below). The consultants also incorporated this content into the workshop they required prospective peer trainers to complete and announced the details of the intervention in consultation meetings held with various management-level employees. Although there were no programmed antecedents or consequences during this phase (i.e., conditions from the feedback phase remained in effect), we included these announcements as a separate phase given previous research showing behavior change may occur when systems-level changes are announced (Luiselli et al., 2009).

\section{Monetary Incentive + Feedback}

This phase included a monetary incentive and feedback. Peer trainers earned $\$ 1.00$ for every hour they were assigned to train if they implemented training with $100 \%$ integrity (i.e., they met criterion) during the observation. That is, if peer trainers were scheduled to train a newly hired DSP for $16 \mathrm{~h}$, they earned $\$ 16$ if they performed the three expected behaviors with $100 \%$ integrity. This monetary amount was determined after consultation with the Finance Department who projected maximum costs to the organization assuming every peer trainer met criterion for every hour trained across a year. Each US dollar earned was added to an individualized tracking spreadsheet that the consultants shared with each peer trainer via email. Peer trainers exchanged their earnings by purchasing items from a gift-giving platform (i.e., Snappy; www.snappy.com) already adopted by the organization. Snappy's website describes itself as a one-stop-shop for corporate gifting needs, such as recognizing employees and colleagues and appreciating customers. There is a slight fee for using the platform. The organization used Snappy for employee recognition and paid a $15 \%$ service fee on top of the actual costs of the gifts purchased. As a result, they asked us to use Snappy for exchanging the monetary incentive. The platform had items that ranged in price; thus, peer trainers could save their earnings for more expensive items. They were required to earn a minimum of $\$ 25$ before making a purchase.

In addition to a monetary incentive, peer trainers received feedback similar to the feedback phase. We also informed the peer trainers about whether they earned the incentive. Because of the COVID-19 pandemic, the organization restricted in-person observations for approximately 4 months; thus, all observations were remote during most of this phase. During in-person observations (i.e., the inperson phase), feedback was provided vocally and immediately following the observation. During remote observations (i.e., remote phase), feedback was provided vocally over the phone. If peer trainers could not be reached via phone, written feedback was provided via email and a phone call could be arranged at a peer trainer's request. During the hybrid phase, when observations were conducted both in-person and remotely, feedback was provided as described above based on the modality of the observation. 


\section{Monetary Incentive + Feedback + Prompt}

In addition to a monetary incentive and feedback, this phase included a supplemental prompt. The prompt consisted of a phone call during which the consultant informed the peer trainer of the DSP trainee's name and the date(s) and time(s) they were scheduled to train. The consultant also reminded the peer trainer of the criterion to earn the monetary incentive. Peer trainers had the opportunity to ask questions and problemsolve training issues they anticipated experiencing. If the peer trainer could not be reached via telephone, a written prompt containing the same information was provided via email.

\section{Interobserver Agreement}

A second observer collected data on peer trainer behavior for $23.6 \%$ of sessions to assess interobserver agreement. An agreement was scored when both observers recorded the peer trainer's behavior in the same way (i.e., as correct or incorrect). A disagreement was scored when both observers did not record the peer trainer's behavior identically. Interobserver agreement was calculated by dividing the number of behaviors with agreement by the number of scored behaviors and multiplying by 100 . Interobserver agreement averaged 93.9\% across all phases (range, 85.4-98.1\%).

\section{Measures}

We measured peer trainer integrity during each observation. Our criterion was that peer trainers would correctly perform the following three behaviors: (1) complete the training checklist while training (thus, it must be visible and immediately available), (2) conduct themselves in a professional manner (e.g., use a pleasant tone of voice, greet colleagues, refrain from expressing frustration, refrain from gossip), and (3) implement the training components specified on the training checklist for the skill being taught. Table 2 contains more information about the target behaviors. We incorporated the first behavior (i.e., peer trainers complete the training checklist while training) given concerns expressed by agency leaders that peer trainers were not referring to the checklist throughout training and were filing incomplete checklists at the conclusion of the training. These behaviors were problematic for the agency for two reasons. First, it is impossible to remember all the skills listed on the training checklist; without referring to the checklist throughout training, it was likely that peer trainers would fail to train some skills. Second, filing incomplete checklists was concerning for state reviewers who audit program quality. If documentation was missing or incomplete, the outcomes of the review would be negatively affected. We incorporated the second behavior (i.e., peer trainers conduct themselves in a professional manner) given unprofessional behaviors we observed, complaints of newly hired staff, and concerns expressed by parents and

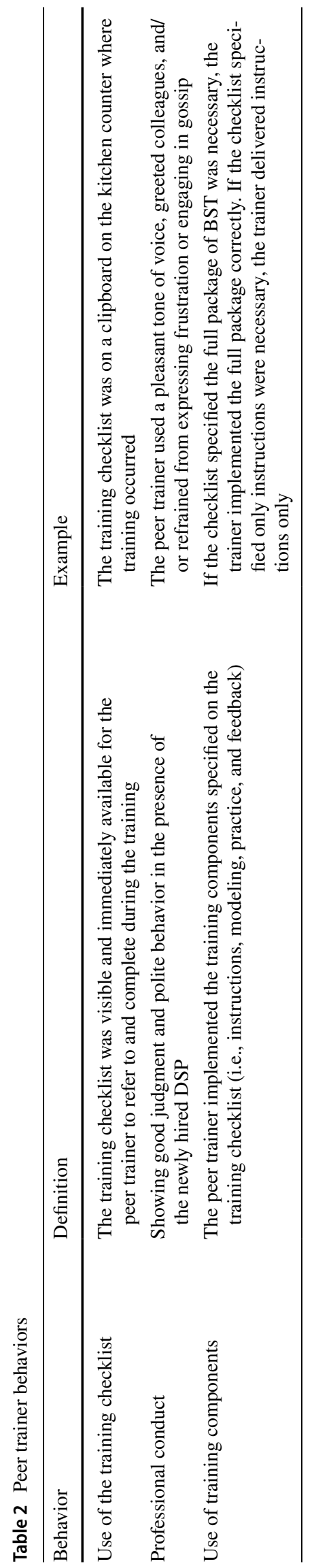


guardians of the consumers served. Finally, we incorporated the third behavior (i.e., peer trainers implement the training components specified on the training checklist for the skill being taught) to ensure trainers used components of BST or the full package of BST as specified on the checklist.

\section{Data Analyses}

A peer trainer behavior was considered correct if the participant accurately performed it as described in Table 2. A behavior was considered incorrect if the participant did not accurately perform the behavior or omitted the behavior. For the purposes of this program description, we summarized the data as the percentage of peer trainers who met our criterion of $100 \%$ correct, which was calculated by dividing the number of peer trainers who met criterion during that observation period (i.e., 2 weeks) by the total number of peer trainers who trained during that observation period and multiplying by 100 . We used visual inspection to analyze these data.

We were also interested in determining the extent to which peer trainers correctly implemented the three peer trainer behaviors. We calculated the mean component integrity of each of the three behaviors for each phase by dividing the number of trainings in which all peer trainers correctly performed the behavior by the total number of opportunities for all peer trainers to engage in that behavior and multiplying by 100 .

\section{Results}

Figure 2 displays the percentage of trainers who met criterion and the number of observations that occurred during the biweekly observations. Table 3 summarizes the component integrity data for each of the peer trainer behaviors across
Table 3 Component integrity analysis for each phase

\begin{tabular}{lrrl}
\hline Phase & \multicolumn{2}{l}{ Percent Component Integrity (\%) } \\
\cline { 2 - 4 } & Checklist & Professionalism & $\begin{array}{l}\text { Training Compo- } \\
\text { nents Implemented }\end{array}$ \\
\hline FB & 10.5 & 79.0 & 55.6 \\
PA & 87.0 & 100.0 & 75.0 \\
MI+F (in-person) & 80.0 & 100.0 & 90.0 \\
BL & 0.0 & 100.0 & 20.0 \\
MI+F (remote) & 44.4 & 91.7 & 68.6 \\
MI+F+P & 81.3 & 90.7 & 88.5 \\
\hline
\end{tabular}

$B L$ baseline, $F B$ feedback, $P A$ program announcement, $M I+F$ monetary incentive + feedback, $M I+F+P$ monetary incentive + feedback + prompt

phases. The number of trainers observed within a 2-week period across the years summarized ranged between one and 12. Thus, some variability in the data can be attributed to the number of trainers observed. During baseline in early September 2019, we conducted observations of peer trainers to determine if integrity remained low as previously identified in the pre-intervention assessment. No trainers met criterion during baseline, suggesting an intervention was necessary. In late September, the CEO provided approval for the consultation team to deliver feedback to peer trainers and indicated he would consider a monetary incentive in the future if feedback did not improve training integrity. During the feedback phase, the percentage of trainers who met criterion was low $(M=5.3 \%$; range, $0-33 \%)$. The component integrity data revealed peer trainers used the training checklist, conducted themselves professionally, and implemented specified training components during $10.5 \%, 79 \%$, and $55.6 \%$ of peer trainings, respectively.
Fig. 2 Percentage of trainers who met criterion during biweekly observations. Note. $\mathrm{BL}=$ baseline, $\mathrm{FB}=$ feedback, $\mathrm{PA}=$ program announcement, $\mathrm{MI}+\mathrm{F}=$ monetary incentive + feedback, $\mathrm{MI}+\mathrm{F}+\mathrm{P}=$ monetary incentive + feedback + prompt. The asterisks (*) indicated biweekly observations where no trainings were observed. Although we calculated the overall percentage of trainers who met criterion for the first observation on $\mathrm{BL}$, the data recording sheets cannot be located and, thus, the number of trainings that occurred is unknown
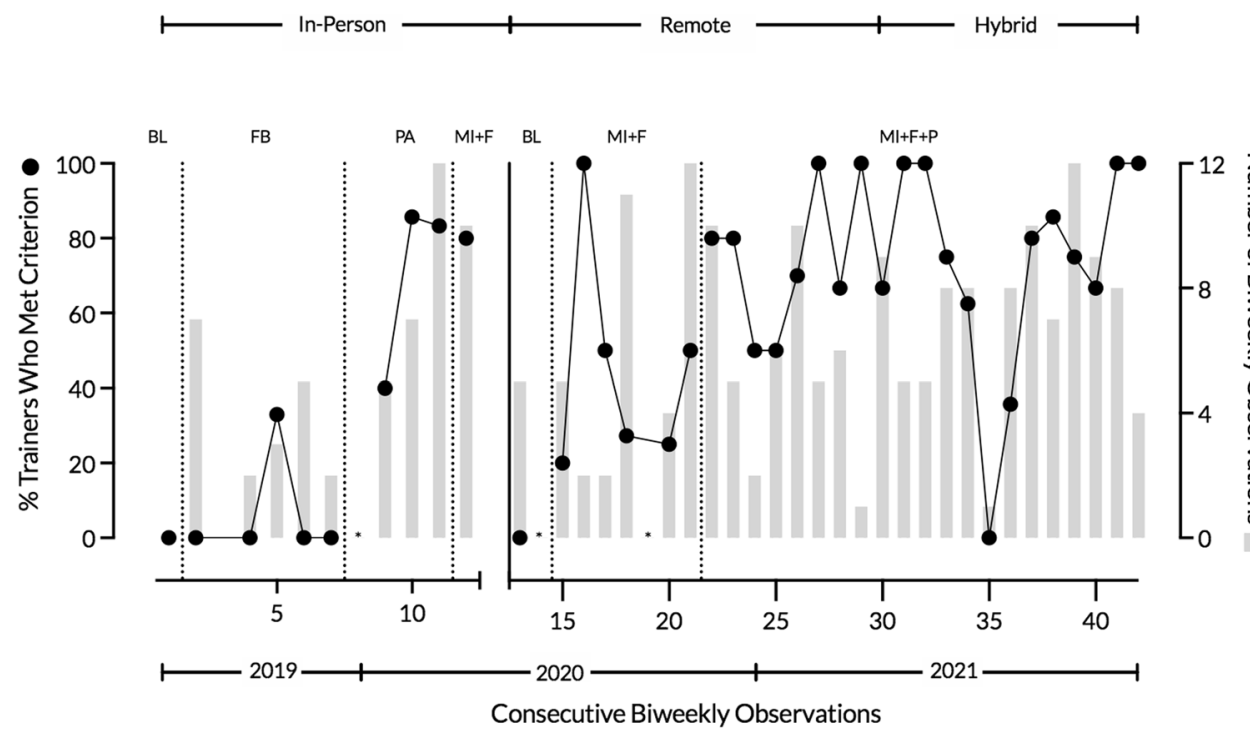
During feedback conversations, multiple peer trainers indicated they were not getting paid to provide training to new staff and that training made their jobs more difficult. Consequently, the consultation team asked the organization to support an intervention with a monetary incentive. In collaboration with the organization's finance and human resources departments, the details of the monetary incentive were finalized, and the program was announced in January and February 2020. The percentage of trainers who met criterion increased when the program was announced despite feedback conditions remaining in effect $(M=75 \%$; range, 40-85.7\%). The component integrity data revealed improvements in the percentage of peer trainers who engaged in each of the three target behaviors. Peer trainers used the training checklist, conducted themselves professionally, and implemented specified training components during $87 \%, 100 \%$, and $75 \%$ of peer trainings, respectively. The monetary incentive plus feedback phase began in late February 2020. Upon introducing a monetary incentive plus feedback, the percentage of trainers who met criterion was $80 \%$. The component integrity data revealed peer trainers used the training checklist, conducted themselves professionally, and implemented specified training components during $80 \%, 100 \%$, and $90 \%$ of peer trainings, respectively. Given safety and staffing concerns associated with the COVID-19 pandemic, the organization placed the intervention, including peer training workshops, on hold for 146 days through July 2020. Thus, there is only one data point in the initial monetary incentive plus feedback phase.

The consultation team was given permission to resume consultation in late July 2020 in a remote format. We collected additional baseline data wherein we observed that no peer trainers implemented training with integrity (i.e., met the criterion). Consequently, we reintroduced the monetary incentive plus feedback (delivered remotely) and the percentage of peer trainers who met criterion increased relative to baseline ( $M=38.9 \%$; range, $20-100 \%)$. The data in this phase were not as high as the first introduction of this intervention. Peer trainers used the training checklist, conducted themselves professionally, and implemented specified training components during $44.4 \%, 91.7 \%$, and $68.6 \%$ of peer trainings, respectively. Because desired levels of performance were not observed, the consultation team incorporated a supplemental prompt, a potential indicated intervention based on the pre-intervention assessment. The inclusion of a prompt for training increased the percentage of peer trainers who met the training criterion with some variability in the data $(M=75.5 \%$, range, $0-100 \%)$. The component integrity data revealed peer trainers used the training checklist, conducted themselves professionally, and implemented specified training components during $81.3 \%, 90.7 \%$, and $88.5 \%$ of peer trainings, respectively.
At the conclusion of data analysis, peer trainers had been awarded \$1994.50; however, the organization assumed only $\$ 150$ in direct costs and $\$ 22.50$ for the Snappy service fee as most peer trainers had not exchanged their earnings for items on www.snappy.com. The reasons peer trainers had not exchanged varied. Some peer trainers no longer worked for the organization and did not exchange their earnings before their departure. Several peer trainers had not yet earned $\$ 25$, the minimum threshold for exchanging their earnings for a reward. Finally, others were eligible to exchange but saved their earnings to purchase more expensive items.

\section{Discussion}

The purpose of this program description was to summarize the results of a systems-level intervention to improve peer trainer integrity within an ongoing consultation arrangement. We adopted procedures from Erath et al. (2021) and expanded them to prepare peer trainers for their role in training new DSPs. A pre-intervention assessment was then conducted to identify potential barriers peer trainers experienced in the workplace. Finally, indicated interventions were implemented in a sequential manner to evaluate their effects on trainer integrity. We adopted a sequential approach to the systems-level intervention to make careful use of limited resources. First, we modified the process for scheduling peer training and delivered feedback to peer trainers following our observations. These indicated interventions did little to produce improvements in the percentage of trainers who conducted training with integrity. Next, we added a monetary incentive and a supplemental prompt and observed increased percentages. These data suggest that the implementation of an assessment-derived intervention at the organizational level can improve the percentage of trainers who conduct training with integrity.

Ensuring peer trainers conduct training with integrity is important for several reasons. Without experiencing highquality, empirically supported training, the performance of newly trained DSPs may be suboptimal, with implications for the quality of services delivered to consumers. For example, research has shown consumer outcomes are affected by the extent to which staff and educators implement interventions, such as behavior plans (e.g., DiGennaro et al., 2007; Wilder et al., 2006) and teaching protocols (e.g., Hirst \& DiGennaro Reed, 2015). Moreover, correct implementation of health protocols by newly hired DSPs decreases the likelihood of staff and consumer injuries or potentially life-threatening risks. Employees in the present organization are regularly responsible for implementing health procedures that can impact a consumer's well-being, such as administering medications, using adaptive equipment, or preparing pureed foods for individualized diets. Thus, implementation errors 
with these or other health procedures may have numerous implications for consumer health. By adopting systems to support peer trainers that ensure high-quality training, such potentially dangerous situations may be prevented.

Our findings contribute to the literature in several ways. First, this program description addresses the limitations of Erath and colleagues $(2020 ; 2021)$ and extends their work to the natural environment. To our knowledge, this is the first study that describes a systematic line of research evaluating the effects of a peer training program using a consultation model within a human services organization. Taken together, this line of work provides information about one systematic approach that organizational leaders or trainers could adopt to improve peer trainer integrity.

Second, our findings provide additional support for the validity of the PDC-HS. Although previous research has demonstrated the utility and validity of the PDC-HS (e.g., Carr et al., 2013), the interventions used in those studies were not implemented across an entire organization for an extended duration or with many employees. The present findings support the long-term adoption of systems-level interventions informed by the PDC-HS. Moreover, much of the PDC-HS research describes an assessment wherein supervisors were interviewed about variables influencing staff behavior. Relatively few studies have used the PDCHS to interview staff about the barriers they are experiencing (see Merritt et al., 2019). Our findings lend support for directly engaging the staff who will experience the intervention to determine the barriers to their performance.

Third, the delivery of a monetary incentive exchangeable through a gift-giving platform was a creative and an affordable way to provide preferred consequences for desirable peer trainer performance. That is, peer trainers did not receive the monetary incentive in their paycheck and instead were able to purchase items from www.snappy.com. The human resources director initially proposed this procedure primarily due to process challenges for paying monetary bonuses for non-exempt staff. The present findings support the use of a monetary incentive delivered in this manner (i.e., exchangeable for various goods on a gift-giving platform). Interestingly, the costs to the organization were minimal, as many peer trainers had not spent their earnings at the time of the writing of this manuscript. Although studies involving monetary incentives and employee token economies have been published in the literature (e.g., Fox et al., 1987; Vergason \& Gravina, 2019), few are used within human services settings. Luiselli et al. (2009) evaluated the effects of an intervention containing a probabilistic financial incentive on chronic absenteeism of human services employees and showed reductions in the percentage of daily staff absences. Thus, the present findings contribute to this small literature and offer an affordable option for organizations to adopt.
We observed variability in responding during the monetary incentive plus feedback and prompt phase. This variability may have been caused by differing numbers of observation sessions conducted during each observation period. That is, the number of observations depended on the number of DSPs hired during that timeframe. For instance, if one observation period included two trainings and one peer trainer did not meet criterion, the percentage of peer trainers to meet criterion would have been $50 \%$. In another week, ten trainings may have occurred and if one peer trainer did not meet criterion, the percentage of peer trainers to meet criterion would have been $90 \%$. In either situation, one peer trainer did not meet criterion, but the overall percentage of trainers who met criterion varied substantially. The observed variability may also have been caused by the regular influx of new peer trainers due to staff turnover, promotions, or professional development opportunities. Thus, the peer trainers whose performance is captured in the data were not a static group, which could have introduced variability in the data.

Integrity improvements were observed during the program announcement phase, despite the monetary incentive not being in place. These data are like the pattern observed in Luiselli et al. (2009) when an informational brochure was distributed to announce the upcoming intervention. The informational brochure used by Luiselli et al. (2009) described the lottery intervention, eligibility, start date, and qualification guidelines. In the present study, the consultants had informal conversations with some peer trainers and supervisory staff about the forthcoming monetary incentive and the qualification guidelines. The start date for the program was not relayed at that time; therefore, peer trainers may have thought the monetary incentive was already in place.

\section{Limitations and Future Research}

There are several limitations worth noting. First, due to the nature of the ongoing consultative relationship and restrictions imposed by the COVID-19 pandemic, there is limited experimental control. Although we returned to baseline for a single observation period, and the percentages were low, our procedures were not designed to assess the effects of the intervention in a tight experimental fashion. Despite this limitation, we were able to replicate our findings within this program evaluation. A future study should adopt a behavior analytic research design, such as a multiple-baseline design across settings or a withdrawal design, to draw causal conclusions about the effects of the packaged intervention on peer trainer integrity. Second, we did not measure consumer outcomes. The primary reason to ensure training is implemented with integrity is to enhance the likelihood that newly hired DSPs will accurately implement procedures, 
particularly health-related protocols, with consumers. We were unable to measure consumer outcomes with the current consultation resources given the number of peer trainers, employees, and programs affected. Future research should evaluate the impact of a peer training program on consumer outcomes. Relatedly, we did not measure whether our efforts were associated with reductions in staff turnover, which would be an ideal secondary outcome. Because the pandemic has caused instability in the workforce for multiple years, we did not conduct this analysis. Additionally, we conducted PDC-HS interviews with four staff, which may not identify representative barriers to performance that all staff members experience. Future research should determine how many assessments are necessary to ensure the representativeness of the results. Finally, our observations relied on iLink Support Technologies® (DiGennaro Reed \& Reed, 2013; Strouse \& DiGennaro Reed, 2021), which is unique to the service setting where consultation occurred. Most organizations do not offer smart-home services with hardware and software of this type, which would make remote observation difficult if not impossible. The extent to which our procedures and findings generalize to other providers is unknown. Thus, future research should examine the external validity of these procedures.

Author Contribution AB: co-designed and executed the program evaluation, assisted with the data analyses, and co-wrote the paper. SR: executed the program evaluation, assisted with the data analyses, and co-wrote the paper. GB: assisted with data collection. FDR: codesigned the program evaluation, supervised all activities, and co-wrote the paper. MS: approved and provided resources for the program evaluation, designed software used for program evaluation. TE: co-designed and executed the program evaluation. MLB: assisted with data collection. All authors approved the final version of the manuscript for submission.

Data Availability All data are available at the Open Science Framework (https://osf.io/j7fdg/).

\section{Declarations}

Ethics Statement All procedures performed in this study were in accordance with the ethical standards of the University of Kansas Human Rights and Protection Protocol and the organization's Human Rights Committee.

Informed Consent Content to participate and publish was obtained from the organization.

Conflict of Interest The authors have no relevant financial or non-financial interests to disclose. The fourth author serves on the Board of Directors for the organization where the research was conducted. The fifth author serves as the organization's Chief Executive Officer and President of the Board.

\section{References}

Anderson, L. L., Humphries, K., McDermott, S., Marks, B., Sisarak, J., \& Larson, S. (2013). The state of the science of health and wellness for adults with intellectual and developmental disabilities. Intellectual Developmental Disabilities, 51(5), 385-398. https:// doi.org/10.1352/1934-9556-51.5.385

Armitage, R., \& Nellums, L. B. (2020). The COVID-19 response must be disability inclusive. Lancet Public Health, 5(5), e257. https:// doi.org/10.1016/S2468-2667(20)30076-1

Braddock, D., Hemp, R., Rizzolo, M. C., Tanis, E. S., Haffer, L., Lulinski, A., \& Wu, J. (2013). State of the states in developmental disabilities 2013: The great recession and its aftermath. Universities of Colorado and Illinois-Chicago.

Carr, J. E., Wilder, D. A., Majdalany, L., Mathisen, D., \& Strain, L. A. (2013). An assessment-based solution to a human-service employee performance problem. Behavior Analysis in Practice, 6(1), 16-32. https://doi.org/10.1007/BF03391789

Centers for Disease Control and Prevention (2020, September 16). Disability and health related conditions. https://www.cdc.gov/ncbddd/ disabilityandhealth/relatedconditions.html

Demchak, M., Kontos, S., \& Neisworth, J. T. (1992). Using a pyramid model to teach behavior management procedures to childcare providers. Topics in Early Childhood Special Education, 12(4), 458-478. https://doi.org/10.1177/027112149201200405

Diament, M. (2020, February 7). Nationally, waiting lists for waiver services growing. https://www.disabilityscoop.com/2020/02/07/ nationally-waiting-lists-waiver-services-growing/27783/

DiGennaro Reed, F. D., Hurst, J. M., \& Howard, V. J. (2013). Empirically supported staff selection, training, and management strategies. In D. D. Reed, F. D. DiGennaro Reed, \& J. K. Luiselli (Eds.), Handbook of crisis intervention and developmental disabilities (pp. 71-85). Springer. https://doi.org/10.1007/ 978-1-4614-6531-7_5

DiGennaro, F. D., Martens, B. K., \& Kleinmann, A. E. (2007). A comparison of performance feedback procedures on teachers' treatment implementation integrity and students' inappropriate behavior in special education classrooms. Journal of Applied Behavior Analysis, 40(3), 447-461. https://doi.org/10.1901/jaba. 2007.40-447

DiGennaro Reed, F. D., \& Reed, D. D. (2013). HomeLink support technologies at community living opportunities. Behavior Analysis in Practice, 6(1), 80-81. https://www.ncbi.nlm.nih.gov/pmc/ articles/PMC3680146/

Erath, T. G., DiGennaro Reed, F. D., \& Blackman, A. L. (2021). Training human service staff to implement behavioral skills training using a video-based intervention. Journal of Applied Behavior Analysis, 54(3), 1251-1264. https://doi.org/10.1002/jaba.827

Erath, T. G., DiGennaro Reed, F. D., Sundermeyer, H. W., Brand, D., Novak, M. D., Harbison, M. J., \& Shears, R. (2020). Enhancing the training integrity of human service staff using pyramidal behavioral skills training. Journal of Applied Behavior Analysis, 53(1), 449-464. https://doi.org/10.1002/jaba.608

Fox, D. K., Hopkins, B. L., \& Anger, W. K. (1987). The long-term effects of a token economy on safety performance in open-pit mining. Journal of Applied Behavior Analysis, 20(3), 215-224. https://doi.org/10.1901/jaba.1987.20-215

Friedman, C. (2019). State utilization of direct support professionals in Medicaid HCBS waivers. Intellectual and Developmental Disabilities, 57(1), 1-13. https://doi.org/10.1352/1934-9556-57.1.1

Gleason, J., Ross, W., Fossi, A., Blonsky, H., Tobias, J., \& Stephens, M. (2021). The devastating impact of COVID-19 on individuals with intellectual disabilities in the United States. Advance online publication. 
Green, C. W., \& Reid, D. H. (1994). A comprehensive evaluation of a train-the-trainers model for training education staff to assemble adaptive switches. Journal of Developmental and Physical Disabilities, 6, 219-238. https://doi.org/10.1007/BF02578412

Harchik, A. E., Anderson, M., Thomson, R., Forde, K., Feinberg, L., Rivest, S., \& Luiselli, J. K. (2001). Evaluation of a participatory competency-based model of staff training in a community habilitative setting. Behavioral Interventions, 16(1), 1-13. https://doi. org/10.1002/bin.77

Harris-Kojetin, L., Sengupta, M., Park-Lee, E., \& Valverde, R. (2013). Long-term care services in the United States: 2013 overview. Vital and Health Statistics, 3(37), 1-107. https://www.cdc.gov/nchs/ data/nsltcp/long_term_care_services_2013.pdf

Hirst, J. M., \& DiGennaro Reed, F. D. (2015). An examination of the effects of feedback accuracy on academic task acquisition in analogue settings. The Psychological Record, 65, 49-65. https://doi. org/10.1007/s40732-014-0087-y

Kansas Department for Aging and Disability Services. (2010). Article 63 - Developmental disabilities - Licensing providers of community services. https://kdads.ks.gov/docs/librariesprovider17/ survey-certification-and-credentialing-commission/hcbs-i-ddlicensure/license-applicants/ks-article-63.pdf?sfvrsn=176539ee_2

Luiselli, J. K. (2015). Staff preparation and performance management: Applied. In F. D. DiGennaro Reed \& D. D. Reed (Eds.), Autism service delivery: Bridging the gap between science and practice (pp. 464-489). Springer.

Luiselli, J. K., DiGennaro Reed, F. D., Christian, W. P., Markowski, A., Rue, H. C., St Amand, C., \& Ryan, C. J. (2009). Effects of an informational brochure, lottery-based financial incentive, and public posting on absenteeism of direct-care human services employees. Behavior Modification, 33(2), 175-181. https://doi. org/10.1177/0145445508320624

Merritt, T. A., DiGennaro Reed, F. D., \& Martinez, C. E. (2019). Using the performance diagnostic checklist-human services to identify an indicated intervention to decrease employee tardiness. Journal of Applied Behavior Analysis, 52(4), 1034-1048. https://doi.org/ 10.1002/jaba.643

Novak, M. D., DiGennaro Reed, F. D., Erath, T. G., Blackman, A. L., Ruby, S. A., \& Pellegrino, A. J. (2019). Evidence-based performance management. Applying behavioral science to support practitioners. Perspectives on Behavior Science, 42(4), 955-972. https://pubmed.ncbi.nlm.nih.gov/31976469/

Parsons, M. F., Rollyson, J. H., \& Reid, D. H. (2013). Teaching practitioners to conduct behavioral skills training: A pyramidal approach for training multiple human service staff. Behavior Analysis in Practice, 6(2), 4-16. https://www.ncbi.nlm.nih.gov/ pmc/articles/PMC5139667/
President's Committee for People with Intellectual Disabilities. (2017). America's direct support workforce crisis: Effects on people with intellectual disabilities, families, communities and the U.S. economy. https://www.acl.gov/sites/default/files/programs/2018-02/ 2017\%20PCPID\%20Full\%20Report_0.PDF

Strouse, M. C., \& DiGennaro Reed, F. D. (2021). Employee turnover and workforce stability. In J. K. Luiselli, R. M. Gardner, F. L. Bird, \& H. Maguire (Eds.), Organizational behavior management $(O B M)$ approaches for intellectual and developmental disabilities (pp. 210-234). Routledge.

Residential Information Systems Project (2020). In-home and residential long-term supports and services for persons with intellectual or developmental disabilities: Status and trends 2017. https://ici-s. umn.edu/files/aCHyYaFjMi/risp_2017

Ryan, A., Taggart, L., Truesdale-Kennedy, M., \& Slevin, E. (2014). Issues in caregiving for older people with intellectual disabilities and their ageing family carers: A review and commentary. International Journal of Older People Nursing, 9(3), 217-226. https:// doi.org/10.1111/opn.12021

Tyler, C. V., Schramm, S. C., Karafa, M., Tang, A. S., \& Jain, A. K. (2011). Chronic disease risks in young adults with autism spectrum disorder: Forewarned is forearmed. American Journal on Intellectual and Developmental Disabilities, 116(5), 371-380. https://pubmed.ncbi.nlm.nih.gov/21905805/

Van den Pol, R. A., Reid, D. H., \& Fuqua, R. W. (1983). Peer training of safety-related skills to institutional staff: Benefits for trainers and trainees. Journal of Applied Behavior Analysis, 16(2), 139156. https://www.ncbi.nlm.nih.gov/pmc/articles/PMC1307872/

Vergason, C. M., \& Gravina, N. E. (2019). Using a guest- and confederate-delivered token economy to increase employee-guest interactions at a zoo. Journal of Applied Behavior Analysis, 53(1), 422-430. https://doi.org/10.1002/jaba.599

Wilder, D. A., Atwell, J., \& Wine, B. (2006). The effects of varying levels of treatment integrity on child compliance during treatment with a three-step prompting procedure. Journal of Applied Behavior Analysis, 39(3), 369-373. https://doi.org/10.1901/jaba. 2006.144-05

Publisher's Note Springer Nature remains neutral with regard to jurisdictional claims in published maps and institutional affiliations.

Author Note GoodLife Innovations, Inc is a long-term collaborative partner with faculty and graduate students from the Department of Applied Behavioral Science at the University of Kansas for applied research that collectively benefits community services serving children and adults with intellectual and developmental disabilities. 